\title{
Hybrid Artificial Fish School Algorithm Based on Mutation Operator for Solving Nonlinear Equations
}

\author{
Yongquan Zhou \\ College of Mathematics and Computer Science \\ Guangxi University for Nationalities \\ Nanning, Guangxi 530006, CHINA \\ yongquanzhou@126.com
}

\author{
Huajuan Huang \\ College of Mathematics and Computer Science \\ Guangxi University for Nationalities \\ Nanning, Guangxi 530006, CHINA \\ hhj-025@163.com
}

\begin{abstract}
To overcome the problems of the classical algorithms for solving nonlinear equations, such as high sensitivity to the initial guess of the solution, poor convergence reliability and can't get all solutions, etc. A hybrid artificial fish swarm algorithm based on mutation operator (HAFSA) is proposed, which combined the advantages of artificial fish school algorithm (AFSA) and the Hooke-Jeeves method. The HAFSA sufficiently exerted the advantages of AFSA such as group search and global convergence, can efficiently overcome the problem of high sensitivity to initial guess, and it also had a high convergence rate and solution precision just because it used the Hooke-Jeeves method which has high local convergence for local search. Besides, mutation operator is embedded to avoid the common defect of premature convergence of the hybrid algorithm. The experimental results show that the proposed hybrid algorithm outperforms the classical numerical methods and the standard artificial fish swarm algorithm significantly in terms of effectiveness and efficiency.
\end{abstract}

Keywords- systems of nonlinear equations; artificial fish school algorithm; Hooke-Jeeves method; hybrid algorithm ; mutation.

\section{INTRODUCTION}

Solving nonlinear equations is often required by various areas of scientific account, such as numerical weather forecast, track design, oil exploration, etc. So far there have existed many reports on solving nonlinear equations. Most of them, however, adopted a conventional numerical method, such as Newton method and its improved form [1]. But the convergence of these methods is high sensitive to the initial guess of the solution for solving nonlinear equations. In other words, it is easily failed to solve nonlinear equations when the initial guess of the solution is ill-suited. However, finding a suited initial guess of the solution is a very difficult problem. So finding a method which does not depend on the initial guess of the solution for solving nonlinear equations is very necessarily [2], [3].

Artificial fish school algorithm (AFSA) [4], [5] is a novel method to search global optimum. It has been used successfully in many fields [6], [7], [8]. It has a good ability of global convergence and is not sensitivity to the initial guess of the solution. Moreover, it is a simple and easy algorithm. Along with the far going applications of AFSA, the shortcoming of the algorithm is found. Although AFSA has a good ability of global convergence, its local convergence rate is lower. On the other hand, as a classical algorithm, the Hooke-Jeeves method [9] has a high local convergence rate, but its global convergence ability is bad and the method is sensitivity to the initial guess of the solution. Analyzing the strongpoint and the shortcoming of both algorithms, we can imagine that if we combine both algorithms in a best way, sufficiently exert the advantages of them, well then the hybrid algorithm will have a high local and global convergence rate and is not sensitivity to the initial guess of the solution. Based on this idea, this paper proposes a hybrid artificial fish school algorithm (HAFSA) which uses the Hooke-Jeeves method as local search behavior of AFSA. However, the hybrid algorithm can improve the local convergence rate, but it will increase the possibility of premature convergence at the same time. So mutation operator is embedded to reduce the possibility. The experimental results show that the proposed hybrid algorithm is superior to classical numerical methods and the standard artificial fish school algorithm in quality and efficiency.

\section{PROBLEM PRESENTATION}

In this paper, we consider the form of nonlinear equations as following (Assuming nonlinear equations contain $\mathrm{n}$ variables $X=\left(x_{1}, x_{2}, \cdots x_{n}\right)$ and $n$ equations $)$ :

$$
\left\{\begin{array}{l}
f_{1}\left(x_{1}, x_{2}, \cdots x_{n}\right)=0 \\
f_{2}\left(x_{1}, x_{2}, \cdots x_{n}\right)=0 \\
\cdots \\
f_{n}\left(x_{1}, x_{2}, \cdots x_{n}\right)=0
\end{array}\right.
$$

Popularly, the nonlinear equations (1) can be transformed the form of optimization problem as following:

$$
\left\{\begin{array}{l}
X=\left(x_{1}, x_{2}, \cdots, x_{n}\right) \\
\min f(X)=\sqrt{\sum_{i=1}^{n} f_{i}^{2}(X)}
\end{array}\right.
$$

In the hybrid algorithm of this paper, the fitness function of 
artificial fish is defined as following:

$$
Y=F\left(X_{i}\right)=\frac{1}{\sqrt{\sum_{k=1}^{n} f_{k}^{2}\left(X_{i}\right)+1}}
$$

From the equation 3, we can know that if $F(X)$ more approaches 1 , the precision of optimum solution is higher. So we define a number approximate 1 as the condition of the end of our algorithm.

\section{HYBRID ALGORITHM}

\section{A. Artificial fish school algorithm}

\section{1) Algorithm principle}

In the process of studying animal's evolvement, people find that animals don't like people who have advanced intelligence, such as complicated logic inferring ability and comprehensive judgments, and their purposes are reached or shown by the simple behavior of individual or colony. AFSA is an optimizing method based on autonomous animate approach, and it is a new thread which searches in the space for global optimum by simulating preying and living movements of fish school. AFSA use the bottom-up development process, that is, first we construct the model of artificial fish (AF), and the individual chooses the fit behavior from the three typical behaviors by itself. In the end, the global optimum is shown by colony or some individual. The algorithm principle [5], [10] is:

$$
\begin{array}{r}
\text { Procedure Artificial Fish school Algorithm } \\
:: \text { AF_init( ): } \\
\text { while the result is not satisfied do } \\
\text { switch (:: AF_evaluate( ) ) } \\
\text { case value1: } \\
:: \text { AF_follow( ); } \\
\text { case value2: } \\
:: \text { AF_swarm( ); } \\
\text { default: } \\
:: \text { AF_prey( ); } \\
\text { end switch } \\
:: \text { AF_move( ); } \\
\text { get_result( ); } \\
\text { end while; } \\
\text { end Artificial Fish_school Algorithm }
\end{array}
$$

\section{2)Defining symbols}

Artificial fish school algorithm (AFSA) is a random search algorithm based on simulating fish swarm behaviors which contain food seeking behavior, swarming behavior and following behavior. It constructs the behaviors of every artificial fish firstly, and then makes the global optimum come true finally based on fish individuals' local searching behaviors. Firstly, we introduce some definitions about AFSA [5], [6].
Assuming $\left\{X_{1}, X_{2} \cdots, X_{N}\right\}$ is a set of artificial fish and $Y=F\left(X_{i}\right)$ represents the fitness function at position $X_{i}$ which is an arbitrary individual fish in the set. Let $d_{i j}=\left\|X_{i}-X_{j}\right\|$ stands for the distance between artificial fish $X_{i}$ and artificial fish $X_{j}$; Visual represents the field of vision of the artificial fish; Step represents the maximum step of the artificial fish moving; $\delta$ represents the density coefficient and try-number stands for the maximum attempt times of the artificial fish moving in its food seeking behavior. The typical behavior of artificial fish is expressed as followings:

\section{3) Algorithm principle}

\section{1) Behavior of food searching}

Assume $X_{i}$ is the artificial fish state at present, and $X_{j}$ is an arbitrary individual fish within $X_{i}$ 's field of view, then we comparing with their fitness function value. If $Y_{j}$ is better than $Y_{i}$, then $X_{i}$ will move one step forward towards the direction of $X_{j}$ within its field of view. Otherwise, $X_{i}$ will randomly move one step within its field of view when it has tried try-number times to find a better position within its field of view but failed. The behavior of food searching can be expressed in Formula 4.

$$
\left\{\begin{array}{cc}
X_{\text {inext }}=X_{i}+\operatorname{Random}(\operatorname{Step}) \frac{X_{j}-X_{i}}{\left\|X_{j}-X_{i}\right\|}, & \text { if } Y_{j}>Y_{i} \\
X_{\text {inext }}=X_{i}+\operatorname{Random}(\operatorname{Step}) & \text { el se }
\end{array}\right.
$$

\section{2) Behavior of swarming}

Assume $X_{i}$ is the artificial fish state at present, $n f$ is the number of its fellow within its field of view, and $X_{c}=\sum X_{j} / n f$. The behavior of swarming can be expressed in Formula 5.

$$
\left\{\begin{array}{cc}
X_{\text {inext }}=X_{i}+\text { Random }(\text { step }) \frac{\left(X_{c}-X_{i}\right)}{\left\|X_{c}-X_{i}\right\|}, & \text { if } Y_{c} / n f>\delta Y_{i} \\
A F-\text { prey }() & \text { el se }
\end{array}\right.
$$

\section{3) Behavior of following}

In the process of the artificial fish swimming, when one fish discovers more food, the other one will move towards the direction. Assume $X_{i}$ is the artificial fish state at present, $X_{\max }$ is the artificial fish which fitness function value is the maximum within its field of view and $Y_{\max }$ is the fitness function of $X_{\max }$. The behavior of following can be expressed in equation 6. 


$$
\left\{\begin{array}{cc}
X_{\text {inext }}=X_{i}+\text { Random }(\text { step }) \frac{\left(X_{\max }-X_{i}\right)}{\left\|X_{\max }-X_{i}\right\|}, & \text { if } Y_{\text {max }} / n f>\delta Y_{i} \\
A F-\operatorname{prey}() & \text { el se }
\end{array}\right.
$$

According to the character of the problem, the artificial fish will select an appropriate behavior to carry out. For example, every artificial fish simulates behaviors of swarming and following, then selects the better one to carry out actually.

\section{B. Hooke-Jeeves method}

As a classical numerical method, Hooke-Jeeves method (HJ) [9] was proposed by Hooke and Jeeves in 1961. HJ is a direct search method and it contains two moving behaviors. The first one is exploration moving behavior and finding advantageous moving direction is its intention. The other one is called pattern moving behavior and speedup along with the advantageous moving direction is its function. HJ has a high convergence rate because of using both behaviors but it is sensitivity to the initial guess of the solution. Furthermore, it commonly just gets local convergence not global as a classical numerical method.

\section{Mutation operator}

Because of using $\mathrm{HJ}$ as local search operator, the hybrid algorithm the paper proposed improves the local convergence rate, but it will increase the possibility of premature convergence at the same time. To overcome this deficiency, mutation operator is embedded to increase the variety of the group. The hybrid algorithm can avoid the common defect of premature convergence in a certain extent through using mutation operator. The behavior of mutation operator can be expressed by the following:

Create a random number $r_{1}$ between 0 with 1 to every artificial fish when the state of the best artificial fish is without change in continuous $k$ time's iteration. If $r_{1}$ is less than the given group mutation probability $P_{1}$, then create other random number $r_{2}$ between 0 with 1 to all dimensions of the corresponding artificial fish. If $r_{2}$ is less than the given individual mutation probability $P_{2}$, update the state of the corresponding dimension by the formula: $x^{\prime}=x+$ rand -0.5 . Where $x^{\prime}$ stands for the new state of the dimension of artificial fish which has been updated; $x$ stands for the state of the dimension of artificial fish which before updated and rand stands for a random number between 0 with 1 .

\section{Process of the hybrid algorithm}

AFSA has a good ability of global convergence, but its local convergence rate is lower. Moreover, the final result AFSA finds commonly just is satisfying area of solution, isn't the accurate. On the other hand, as a classical numerical method, Hooke-Jeeves method has a high local convergence rate, but its global convergence ability is bad and the method is sensitivity to the initial guess of the solution. If we can structure the hybrid algorithm which contains AFSA and Hooke-Jeeves method rationally, the hybrid algorithm will be a perfect global optimum algorithm. The paper gives the process of the algorithm by the following steps:

Step 1: Initialize some variables, N- number of fishes; Visual - the field of vision of the fish; Step - the maximum step of the fish moving; $\delta$ - the density coefficient; trynumber; $P_{1}$ - group mutation probability; $P_{2}$ - individual mutation probability; $k$ - conditional number for executing mutation operator; $k_{t}$ - number of iteration of Hooke-Jeeves method; randomly generate the given number of artificial fishes.

Step 2: Compare the food concentration of every fish, record down the best one which are signed by $\max Y$ and $\max X$.

Step 3: The fishes start optimization process by its several behaviors.

Step 4: After each optimization action, compare the food concentration of every fish with $\max Y$. If $Y_{u}>\max Y$, then $Y_{u} \rightarrow \max Y$, and $X_{u} \rightarrow \max X$.

Step 5: Use $\max X$ as the initial of the solution to execute Hooke-Jeeves method. Record down the best solution besty which Hooke-Jeeves method search when the algorithm meets the end condition of Hooke-Jeeves method. Then exit operation of the Hooke-Jeeves method. If besty meets the end condition of the hybrid algorithm, end the whole algorithm, otherwise go to Step 6.

Step 6: Execute the mutation operator when satisfy the condition of mutation, otherwise go to Step 8 .

Step 7: After executing the mutation operator, compare the food concentration of every fish with the best status of the fishes which has been recorded down. If the new is better than the old, then use the new updating the old.

Step 8: If the best status of the fishes which has been recorded down meets the condition of the end, then output the optimization result and end the algorithm. Otherwise go to Step 3.

\section{NUMERICAL EXPERIMENTS}

In order to evaluate the effectiveness and efficiency of the hybrid the proposed algorithm (HAFSA) for solving nonlinear equations, numerical experiments have been carried out on a number of problems. HAFSA is compared with the result of [10] which contains classical numerical methods and the standard artificial fish swarm algorithm (SAFSA). Parameters of HAFSA are defined as following: $\mathrm{N}=100$, Visual $=2.5$, Step $=0.3, \delta=0.618$, try-number $=6, P_{1}=0.8, P_{2}=0.001$, $k=6, k_{t}=60$. As HAFSA has stochastic performance, 10 computations are simulated with the same as [10], in order to compare the different optimization approaches for solving nonlinear equations. The statistic performance comparisons of the several algorithms are shown in following three tables. 
Example 1 Nonlinear equations

$$
\left\{\begin{array}{l}
x_{2}+\sin x_{1}=2.2 \\
4 x_{1}+x_{2}^{2}=7
\end{array}\right.
$$

Result produced by the classical method from [10] and by the HAFSA to solve equations 7 to the given preciseness ( $F=0.999999999999999)$. (See Table.1)

From Table 1, we see that HAFSA performs consistently better than the results of [10]. It could find more accurate solution within the given less error. The corresponding error curve for HAFSA is shown in Figure1, which shows that HAFSA has fast convergence speed.

Example 2 Nonlinear equations

$$
\left\{\begin{array}{l}
4 x_{1}^{2}+x_{2}^{2}+2 x_{1} x_{2}-x_{2}=2 \\
2 x_{1}^{2}+3 x_{1} x_{2}+x_{2}^{2}=3
\end{array}\right.
$$

Result produced by the classical method from [10] and by the HAFSA to solve equations 8 to the given preciseness ( $F=0.99999999999999)$. (See Table 2)
From Table 2, we see that HAFSA not only performs better than the classical methods and AFSA, further more, it gets other solution which the classical methods can not find. Although AFSA also get the other solution, the convergence rate of HAFSA is better than the AFSA. From Figure 2 and Figure 3, we get the information that the convergence rate of HAFSA is very fast.

Example 3 Nonlinear equations

$$
\left\{\begin{array}{l}
x_{1} \ln 3+3 \ln x_{2}=\ln 5184 \\
x_{1} \ln 3 x_{2}+3 \ln x_{1} x_{2}+x_{2} \ln 3 x_{1}=\ln 62208
\end{array}\right.
$$

Result produced by the classical method from [10] and by the HAFSA to solve equations 9 to the given preciseness ( $F=0.999999999999)$. (See Table 3)

From Table 3, we see that HAFSA is a good method for solving transcendental equation. It not only has a high convergence rate, but also gets more accurate solution. From Figure 4 and Figure 5, we can conclude that the HAFSA convergence rate and stability is good.

TABLE 1. Comparisons among ANN, Analytic Algorithm, AFSA and HAFSA

\begin{tabular}{|l|c|c|c|c|}
\hline \multicolumn{1}{|c|}{ Method } & $\begin{array}{c}\text { Averaged } \\
\text { iterations }\end{array}$ & $x_{1}$ & $x_{2}$ & $F(X)$ \\
\hline $\begin{array}{l}\text { Couplin } \\
\text { ANN [10] }\end{array}$ & - & 1.44622 & 1.10887 & 0.90915 \\
\hline $\begin{array}{l}\text { Analytic } \\
\text { algorithm [10] }\end{array}$ & - & 1.37890 & 1.21830 & 0.99984 \\
\hline AFSA [10] & 367 & 1.37926 & 1.21736 & 0.99863 \\
\hline HAFSA & 17 & 1.37890267729960 & 1.21835515790824 & 0.999999999999999 \\
\hline
\end{tabular}

TABLE 2: Comparisons among ANN, Newton Method, AFSA and HAFSA

\begin{tabular}{|c|c|c|c|c|}
\hline Method & $\begin{array}{c}\text { Averaged } \\
\text { iterations }\end{array}$ & $x_{1}$ & $x_{2}$ & $F(X)$ \\
\hline Coupling ANN [10] & - & 0.49953 & 1.00430 & 0.98622 \\
\hline Newton Method[10] & - & 0.5 & 1.0 & 1 \\
\hline $\begin{array}{c}\text { AFSA [10] } \\
\text { (first solution) }\end{array}$ & 157 & 0.50001 & 0.99991 & 0.99972 \\
\hline $\begin{array}{c}\text { HAFSA } \\
\text { (first solution) }\end{array}$ & 5 & 0.50000000000000 & 1.000000000000000 & 0.999999999999999 \\
\hline $\begin{array}{c}\text { AFSA [10] } \\
\text { (second solution) }\end{array}$ & 157 & -0.42772 & 2.38677 & 0.99992 \\
\hline $\begin{array}{c}\text { HAFSA } \\
\text { (second solution) }\end{array}$ & 4 & 0.42772618026912 & 2.38679339064919 & 1.000000000000000 \\
\hline
\end{tabular}

TABLE 3: Comparisons among MATLAB Method, AFSA and HAFSA

\begin{tabular}{|c|c|c|c|c|}
\hline Method & $\begin{array}{c}\text { Averaged } \\
\text { iterations }\end{array}$ & $x_{1}$ & $x_{2}$ & $F(X)$ \\
\hline MATLAB (fslove) & - & 0.43213 & 18.14101 & 0.37156 \\
\hline $\begin{array}{c}\text { AFSA [10] } \\
\text { (first solution) }\end{array}$ & 84 & 0.43221 & 14.77369 & 0.99979 \\
\hline $\begin{array}{c}\text { HAFSA } \\
\text { (first solution) }\end{array}$ & 35 & 0.43220720836422 & 14.77349498579719 & 0.9999999999999 \\
\hline $\begin{array}{c}\text { AFSA [10] } \\
\text { (second solution) }\end{array}$ & 84 & 9.52057 & 0.52965 & 0.99938 \\
\hline $\begin{array}{c}\text { HAFSA } \\
\text { (second solution) }\end{array}$ & 6 & 9.52106912465366 & 0.52964740476515 & 1.000000000000000 \\
\hline
\end{tabular}




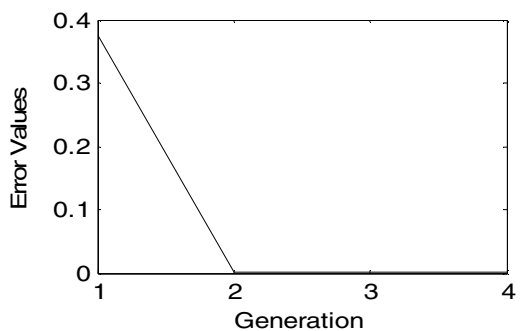

Figure 1. The error curve of the HAFSA for finding the roots of equations 7

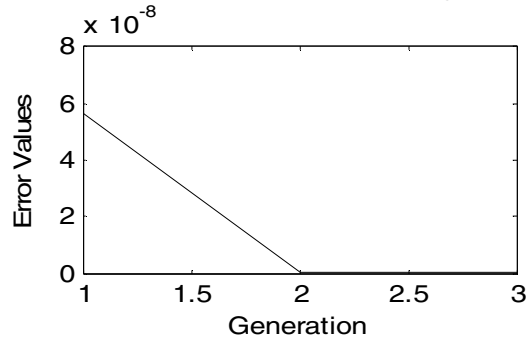

Figure 2. The error of the HAFSA for finding the first roots of equations 8

\section{CONCLUSIONS}

In this paper, nonlinear equations solving by a new algorithm, HAFSA, is proposed. HAFSA is a hybrid of $\mathrm{HJ}$ and AFSA based on mutation operator, it has the advantages of the two at the same time. With the above performance, HAFSA is good algorithm to solving nonlinear equations. It has solved the problem of the classical algorithms for solving nonlinear equations and has increased convergence rate. The results of numerical experiments demonstrate that the HAFSA performs much better than the classical algorithm and AFSA for solving nonlinear equations. It is more efficient and robust and is also very simple and easy to implement.

\section{ACKNOWLEDGMENT}

This work is supported by Grants 60461001 from NSF of China and the project Supported by Grants 0832082 from Guangxi Science Foundation

\section{REFERENCES}

[1] X. L. Huang, Z.G. Zeng, Y.N. Ma, Nonlinear Numerical Analysis. Wuhan: Wuhan University Press, 2002

[2] C. Yang, G.X. Hong, A recommendable method to solve nonlinear algebraic, Flight Dynamics, vol. 15, no. 2, pp. 573-576, 1997.

[3] X. B. Hu, S.F. Wu, J.Jiang," A new method based on genetic algorithms for resolving algebraic equations", Control Theory and Application, vol. 19, no. 4, 2002,pp. 573-576.

[4] X.Y. Tu. Artificial Animals for Computer Animation: Biomechanics, Locomotion, erception, and Behavior, Dissertation for doctor degree, 1999.

[5] X. L. Li, Z. J. Shao, J.X. Qian, "An optimizing method based on autonomous animate: fish swarm algorithm", System Engineering Theory and Practice, vol. 22, no. 11, 2002,pp. 32-38.

[6] C.R. Wang, C.L. Zhou, J.W. Ma, "An improved artificial fish swarm algorithm and its application in feedforward neural networks", Proc. of the Fourth Int. Conf. on Machine Learning and Cybernetics, 2005,pp. 28902894.

[7] X.L. Li, F. Lu, G.H. Tian, J.X. Qian, "Applications of artificial fish school algorithm in combinatorial optimization problems", Journal of Shandong University(Engineering Science), vol. 34, no. 5, 2004, pp. 64-67.

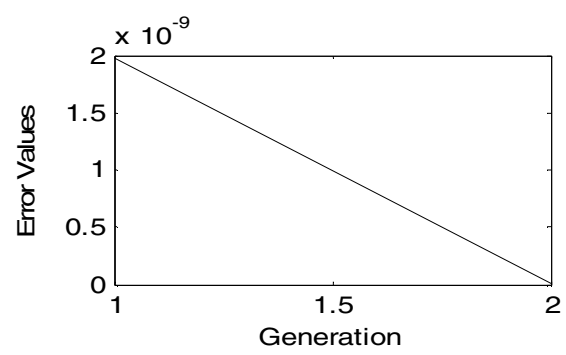

Figure 3. The error of the HAFSA for finding the second roots of equations 8

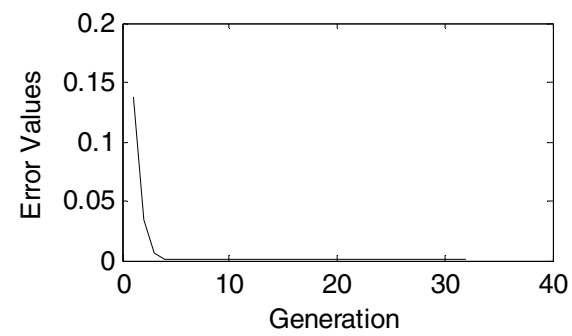

Figure 4. The error of the HAFSA for finding the first roots of equations 9

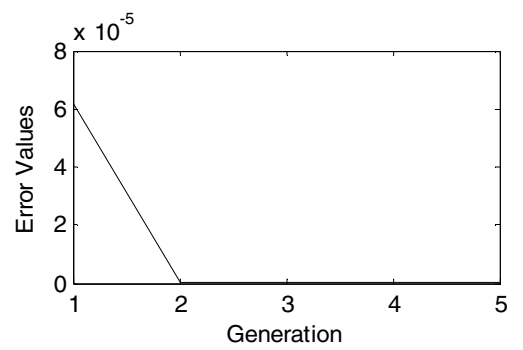

Figure 5. The error of the HAFSA for finding the second roots of equations 9

[8] X.L. Li, S.H. Feng, J.X. Qian, F. Lu, "Parameter tuning method of robust PID controller based on artificial fish school algorithm", Information and Control, vol. 33, no. 1, 2004,pp. 112-115.

[9] H.W. Tang, Practicality optimization method, Dalian: Dalian Science University Press, 1994.

[10] D. D. Wang, Y. Q. Zhou. "Artificial fish-swarm algorithm for solving nonlinear equations", Application Research of Computers, vol. 24, no. 6, 2007, pp. 222-224. 